\title{
Factors associated with SARS-CoV-2 positivity in 20 homeless shelters in Toronto, Canada, from April to July 2020: a repeated cross-sectional study
}

\author{
Tara Kiran MD MSc, Amy Craig-Neil MSc, Paul Das MD MSc, Joel Lockwood MD, Ri Wang MMath, \\ Nikki Nathanielsz BSc, Esther Rosenthal MD, Carolyn Snider MD MPH, Stephen W. Hwang MD MPH
}

\section{Abstract}

Background: It is unclear what the best strategy is for detecting severe acute respiratory syndrome coronavirus 2 (SARSCoV-2) among residents of homeless shelters and what individual factors are associated with testing positive for the virus. We sought to evaluate factors associated with testing positive for SARS-CoV-2 among residents of homeless shelters and to evaluate positivity rates in shelters where testing was conducted in response to coronavirus disease 2019 (COVID-19) outbreaks or for surveillance.

Methods: We conducted a retrospective chart audit to obtain repeated cross-sectional data from outreach testing done at homeless shelters between Apr. 1 and July 31, 2020, in Toronto, Ontario, Canada. We compared the SARS-CoV-2 positivity rate for shelters where testing was conducted because of an outbreak (at least 1 known case) with those tested for surveillance (no known cases). A patient-level analysis evaluated differences in demographic, health and behavioural characteristics of residents who did and did not test positive for SARS-CoV-2 at shelters with at least 2 positive cases.

Results: One thousand nasopharyngeal swabs were done on 872 unique residents at 20 shelter locations. Among the 504 tests done in outbreak settings, 69 (14\%) were positive for SARS-CoV-2 and $1(0.2 \%)$ was indeterminate. Among the 496 tests done for surveillance, $11(2 \%)$ were positive and none were indeterminate. Shelter residents who tested positive for SARS-CoV-2 were significantly less likely to have a health insurance card $(54 \% \mathrm{v} .72 \%, p=0.03)$ or to have visited another shelter in the last 14 days ( $0 \%$ v. $18 \%, p<0.01)$. There was no association between SARS-CoV-2 positivity and medical history or symptoms.

Interpretation: Our findings support testing of asymptomatic shelter residents for SARS-CoV-2 when a positive case is identified at the same shelter. Surveillance testing when there are no known positive cases may detect outbreaks, but further research should identify efficient strategies given scarce testing resources.

$\mathrm{n}$ any given night, more than half a million people in the United States ${ }^{1}$ and more than 35000 in Can$\mathrm{ada}^{2}$ experience homelessness. Homelessness has always been associated with poor health outcomes, ${ }^{3}$ but its risks to health have only been heightened by coronavirus disease 2019 (COVID-19), with consequences extending to the broader community. People experiencing homelessness are at increased risk of acquiring severe acute respiratory syndrome coronavirus 2 (SARS-CoV-2) infections and spreading the virus to others., ${ }^{4,5}$

Many people experiencing homelessness stay in congregate living settings such as shelters, where it is difficult to practise social distancing. Others live rough, on the street or in encampments, and do not have access to basic hygiene supplies or showering facilities. In any of these scenarios, selfisolation is not possible. People who experience homelessness also have higher rates of chronic conditions such as diabetes and hypertension, which puts them at increased risk of complications if they acquire the infection. ${ }^{3,6}$ Recent data from Boston suggest that people experiencing homelessness have a higher prevalence of SARS-CoV-2 positivity $^{7}$ and more severe

Competing interests: Tara Kiran is the Fidani Chair in Improvement and Innovation at the University of Toronto. She is supported as a clinician scientist by the Department of Family and Community Medicine at the University of Toronto and at St. Michael's Hospital. At the time of the study, she was also supported by the Canadian Institutes of Health Research and Health Quality Ontario as an embedded clinician researcher. No other competing interests were declared.

This article has been peer reviewed.

Correspondence to: Tara Kiran, tara.kiran@utoronto.ca

CMAJ Open 2021. DOI:10.9778/cmajo.20200253 
COVID-19 disease ${ }^{8}$ than people who are housed. A preprint modelling study has predicted substantial deaths in the short term because of COVID-19 among the homeless population in the US. ${ }^{9}$

Strategies for testing and follow-up care affect the potential spread of the virus among people experiencing homelessness. ${ }^{5,10}$ Outreach testing early in the pandemic confirmed high rates of SARS-CoV-2 among people experiencing homelessness who were asymptomatic but residing in a shelter with a known case. ${ }^{7}$ However, positivity rates have been variable when testing was done in the absence of a known case. ${ }^{11,12}$ It is still unclear what the best strategy is for detecting SARS-CoV-2 among people experiencing homelessness who live in a congregate setting. It is also unclear whether, among people experiencing homelessness, what individual demographic or health characteristics are associated with acquiring SARS-CoV-2, as these may be different from those in the general population.

Our institution began conducting mobile outreach testing for SARS-CoV-2 in homeless shelters in Toronto, Ontario, about 1 month after the World Health Organization declared the global pandemic. We describe the results of mobile outreach testing at 20 different shelter locations over a 3-month period. We conducted a descriptive comparison of positivity rates among shelters where testing was conducted because of an outbreak (i.e., at least 1 known COVID-19 case at the shelter) or for surveillance (i.e., no known COVID-19 cases). We also sought to assess whether there was any association between individual-level demographic, health or behavioural characteristics and SARS-CoV-2 positivity.

\section{Methods}

\section{Context and setting}

Toronto is Canada's largest city, with a total population of 2956024 in 2018. ${ }^{13}$ Toronto's homeless population was estimated at 8715 in 2018, with about $80 \%$ living in the city's 75 shelter sites, ${ }^{14}$ most of which are located in the downtown core.

St. Michael's Hospital is located in Toronto's downtown core. The hospital has a history of serving people experiencing homelessness and has developed partnerships to provide care at many of the nearby shelters. All permanent residents of Ontario have health insurance via the Ontario Health Insurance Plan. Medically necessary physician visits, hospital services and laboratory tests are fully covered and free at the point of care, including testing for SARS-CoV-2. During the COVID-19 pandemic, nonpermanent residents without provincial health insurance also have free access to testing for SARS-CoV-2. ${ }^{15}$

On Mar. 16, 2020, St. Michael's Hospital opened one of Ontario's COVID-19 Assessment Centres (CACs). These centres were established across the province to facilitate quick and easy access to free testing for SARS-CoV-2. Testing criteria have evolved over time with an increase in testing supplies and laboratory capacity. Initially, testing criteria were restrictive. However, beginning Mar. 18, 2020, testing was made available to symptomatic people who either had an occupation within an at-risk setting or who were residents in a congregate setting, such as a homeless shelter. Testing criteria were slowly expanded, and by June 2, 2020, testing was available to any individual, regardless of contact history, symptoms or living situation. Restrictions on testing were reintroduced in the fall, when resources were stretched with the onset of the second wave of COVID-19. ${ }^{16}$

Early in the pandemic, there were concerns about the transmission and spread of SARS-CoV-2 in homeless shelters. In response, the St. Michael's Hospital CAC team, in partnership with Sherbourne Health, began conducting mobile outreach testing to shelters in the downtown core. Initially, the CAC conducted testing in shelters on request from the local public health unit in response to an outbreak. Outbreaks were defined as one or more cases of COVID-19 in a congregate living facility. ${ }^{17}$

As community case counts decreased at the end of the first wave, the focus shifted to identifying high-risk settings with asymptomatic transmission. As a result, the health region began coordinating surveillance testing in shelters and directed CACs in the region to perform testing in specific shelters, regardless of whether or not there was an outbreak. Shelter residents, either independently or on the advice of shelter staff or medical professionals, could also choose to visit a CAC site or emergency department for testing. During this time, staff also supported shelter residents to move into hotels that were rented and repurposed by the city in an effort to reduce crowding and the related risk of SARS$\mathrm{CoV}-2$ transmission. ${ }^{18}$ Mobile outreach testing was also conducted at some of these hotel sites. In all cases, testing was optional for shelter residents.

\section{Study design and population}

We conducted a retrospective audit of records from all shelter residents tested for SARS-CoV-2 by the St. Michael's Hospital CAC mobile outreach team. Mobile outreach testing was done by the team at 20 unique shelter locations between Apr. 1 and July 31, 2020, at the direction of the local public health unit and the regional health authority; these agencies made a decision to pause testing in August 2020.

We analyzed data from shelter residents for all instances of mobile outreach testing done by the St. Michael's team. We excluded test results from shelter staff. We chose to focus on mobile outreach testing results and did not include charts from the main CAC site because of differences in population, testing criteria and data collection. Testing for SARS-CoV-2 was done using nasopharyngeal swabs and was performed by a physician, nurse practitioner or registered nurse.

\section{Data collection}

The CAC collected data as part of its routine practice and not purposely for research. The outreach team collected age, sex and health insurance number at registration for all shelter residents who were tested. In some cases, the health insurance number was not available either because the resident did not have provincial health insurance (e.g., undocumented resident or refugee claimant with Interim Federal Health Program 
coverage) or did not have their health insurance card (e.g., card lost or misplaced), and the information was not available in the hospital registration system.

When sufficient staff were available for outreach, the CAC mobile outreach team also collected more detailed patient information on a standardized paper form (Appendix 1, available at www.cmajopen.ca/content/9/1/E302/suppl/DC1) that was later scanned into the electronic medical records at St. Michael's Hospital. Staff asked patients questions related to race, symptoms, past medical history and shelter use in the previous 14 days. The CAC team documented all SARSCoV-2 test results in an electronic spreadsheet. The CAC leadership collected information on the number of people eligible for testing at a given shelter and whether testing was for outbreak or surveillance.

A team of 3 research staff, trained by the study coordinator, extracted data for the study. Research staff used the electronic spreadsheet to identify which residents were tested, when and where residents were tested and the test result; staff then extracted data from the scanned form and registration information stored in the hospital electronic record system. Staff extracted data using a Microsoft Access form to help ensure standardization and to reduce error. Research staff initially reviewed the same 20 charts independently, then met to review results; this initial exercise revealed consistency with data extraction. During ongoing data abstraction, uncertainties in the charts were reviewed with another member of the team and consensus was reached.

\section{Statistical analysis}

We performed a shelter-level descriptive analysis, assessing the number of shelter residents eligible for testing and the number tested, as well as the testing date and results. We categorized shelters as either outbreak or surveillance depending on the purpose of outreach testing. We calculated the shelter positivity rate and plotted this over time in relation to local COVID-19 case counts from the City of Toronto website (www.toronto.ca/home/covid-19/covid-19-latest-city-of-toronto -news/covid-19-status-of-cases-in-toronto/).

We also performed a patient-level analysis and assessed the demographic, health and behavioural characteristics of the shelter residents who were tested. For the subset of shelters where there was more than 1 resident who tested positive for SARS-CoV-2, we compared the characteristics of residents who did and did not test positive. For residents who were tested more than once, we categorized them as testing positive if any of their results came back positive. For residents who filled more than 1 demographic sheet, we used the demographic responses associated with the positive test or the earliest collected complete response.

We used a fixed effect logistic regression model to test whether differences in characteristics were statistically significant after adjustment for the shelter location. Data from different days for the same shelter were pooled together and treated as coming from the same cluster. The overall $p$ value for each characteristic was calculated using a likelihood ratio test, comparing a model with and without the characteristic, and adjusting for shelter location. We did not have data on race, health and behavioural characteristics for residents who did not complete a form and treated this as missing data in the analysis. We decided not to perform further multivariable regression analyses because of these missing data. We used Microsoft Access to collect chart audit data and $\mathrm{R}$ version 4.0 for analyses.

\section{Ethics approval}

The study was reviewed and approved by the Unity Health Toronto Research Ethics Board (REB no. 20-132).

\section{Results}

We conducted mobile outreach testing at 20 unique shelter locations on 25 different dates; 4 shelters were tested more than once (Appendix 2, available at www.cmajopen.ca/ content/9/1/E302/suppl/DC1). We conducted testing for a suspected outbreak between Apr. 23 and June 1, 2020, for 430 residents at 6 shelters on 10 different dates; between $40 \%$ and $94 \%$ of those living at the shelters agreed to be tested. We performed surveillance testing between June 9 and July 23, 2020, for 442 residents at 17 shelters over 15 dates, including 3 hotel sites; between $15 \%$ and $86 \%$ of those living at the shelters agreed to be tested. Most shelters where testing was conducted for a suspected outbreak served only men, and those where testing was conducted for surveillance were more varied in the population served.

\section{Individual-level characteristics}

The outreach team conducted 1000 tests for 872 unique shelter residents (504 tests in outbreak settings and 496 tests for surveillance). The demographic and health characteristics of unique shelter residents tested are summarized in Table 1. The mean age of residents was 46, 709 (81.7\%) were men and $589(67.6 \%)$ were able to provide a valid provincial health insurance card.

More detailed demographic information was available for a subset of 348 residents (307 from outbreak and 41 from surveillance testing). Among this group, 134 (40.0\%) residents were White, $174(52.0 \%)$ were racialized and 27 $(8.0 \%)$ chose not to disclose their racial identity. Regarding health status, $248(81.6 \%)$ reported a health condition, with approximately one-quarter saying they had a mental health condition $(n=82)$, almost a third disclosing a substance use disorder $(n=93)$ and over half stating they smoked cigarettes $(n=168)$. Only $32(9.7 \%)$ residents in this subset reported any symptoms of COVID-19, with the most common symptom being a cough; $41(11.8 \%)$ reported visiting another shelter in the last 14 days.

\section{Shelter-level analysis}

Among the 504 tests done in outbreak settings, 69 (13.7\%) were positive and $1(0.2 \%)$ was indeterminate. In subsequent analysis, we excluded the indeterminate result. Among the 496 tests done for surveillance, $11(2.2 \%)$ were positive and none were indeterminate. 
Table 1: Sociodemographic and health characteristics, shelter use and symptom prevalence for residents tested during mobile outreach

\begin{tabular}{|c|c|}
\hline Characteristic & $\begin{array}{c}\text { No. }(\%) \text { of shelter } \\
\text { residents }{ }^{\star} \dagger \\
n=872\end{array}$ \\
\hline \multicolumn{2}{|l|}{ Age, yr } \\
\hline $0-15$ & $38(4.4)$ \\
\hline $16-24$ & $37(4.2)$ \\
\hline $25-49$ & $417(47.9)$ \\
\hline $50-64$ & $277(31.8)$ \\
\hline$>65$ & $102(11.7)$ \\
\hline Age, mean $\pm S D, y r$ & $45.8 \pm 16.3$ \\
\hline Sex, female & $159(18.3)$ \\
\hline $\begin{array}{l}\text { Provincial health insurance number } \\
\text { available }\end{array}$ & $589(67.6)$ \\
\hline \multicolumn{2}{|l|}{ Race } \\
\hline Black & $77(23.0)$ \\
\hline East or Southeast Asian & $23(6.9)$ \\
\hline Latino & $9(2.7)$ \\
\hline Middle Eastern & $9(2.7)$ \\
\hline South Asian & $22(6.6)$ \\
\hline White & $134(40.0)$ \\
\hline Other & $34(10.1)$ \\
\hline Prefer not to disclose & $27(8.0)$ \\
\hline \multicolumn{2}{|l|}{ Past medical history } \\
\hline Any chronic condition & $248(81.6)$ \\
\hline CV disease & $17(5.6)$ \\
\hline Chronic lung disease & $19(6.2)$ \\
\hline HIV & $9(3.0)$ \\
\hline Diabetes & $26(8.6)$ \\
\hline Current smoker & 168 (55.3) \\
\hline Mental health diagnosis & $82(27.0)$ \\
\hline Substance use & $93(30.6)$ \\
\hline Other & $38(12.5)$ \\
\hline Prefer not to disclose & $25(8.2)$ \\
\hline \multicolumn{2}{|l|}{ Symptoms } \\
\hline Any symptoms & $32(9.7)$ \\
\hline Cough & $20(6.0)$ \\
\hline Shortness of breath $\ddagger$ & $-(<2.0)$ \\
\hline Fever & $0(0.0)$ \\
\hline Other & $12(3.6)$ \\
\hline Visited another shelter in last 14 days & $41(11.8)$ \\
\hline \multicolumn{2}{|c|}{$\begin{array}{l}\text { Note: } \mathrm{CV}=\text { cardiovascular, } \mathrm{SD}=\text { standard deviation } \\
\text { *Unless indicated otherwise. } \\
\text { † } 871 \text { residents reported age and } 868 \text { reported sex. Race }(n=335) \text {, past } \\
\text { medical history }(n=304) \text {, symptoms }(n=331) \text { and whether the individual visited } \\
\text { another shelter }(n=348) \text { were based on demographic information collected for } \\
\text { only } 348 \text { respondents. } \\
\text { †Exact number suppressed for confidentiality. }\end{array}$} \\
\hline
\end{tabular}

Figure 1 summarizes the positivity rate by shelter and testing date in relation to the total new number of COVID19 cases in Toronto. Among the 10 testing dates where testing was performed in response to an outbreak, 2 found no positive cases and 1 found a single positive case; the positivity rate for the remaining dates ranged from $4 \%$ to $33 \%$. Only 1 of 17 shelters tested for surveillance had any positive cases. Outbreak testing occurred between April and early June, the months with the highest case counts in Toronto in the study period. In 6 of 10 instances of outbreak testing, the positivity rate was the same or higher than the average positivity rate in Toronto (Appendix 3, available at www.cmajopen. $\mathrm{ca} /$ content/9/1/E302/suppl/DC1).

\section{Individual-level analysis}

We compared the demographic characteristics between shelter residents who tested positive and those who tested negative for SARS-CoV-2. We included data from the 8 testing dates at 4 shelter locations where more than 1 resident tested positive (Table 2). Residents who tested positive were significantly less likely to have a provincial health insurance card $(53.9 \%$ v. $72.4 \%, p=0.03)$ and significantly less likely to have visited another shelter in the last 14 days $(0.0 \%$ v. $17.7 \%$, $p<0.01)$ compared with those who tested negative. Residents who tested positive also had a higher mean age compared with those who tested negative ( 48.3 v. $45.5, p=0.08)$ and a higher proportion were racialized $(61.9 \%$ v. $47.6 \%, p=0.20)$, but these differences were not statistically significant. There were no differences between groups related to the presence of any medical condition or any symptom.

\section{Interpretation}

We conducted 1000 tests for SARS-CoV-2 by mobile outreach testing at 20 shelter locations in Canada's largest city between Apr. 23 and July 23, 2020, during which time the number of new daily cases of COVID-19 in the city dropped from 237 to 31 . About half of the tests were done because of a suspected outbreak and half for surveillance, with the former coinciding with higher numbers of new cases in the city. We found that $14 \%$ of tests done in an outbreak setting were positive compared with $2 \%$ done for surveillance. We found no association between positivity for SARS-CoV-2 and the presence of any medical history or any symptoms. Shelter residents who tested positive were significantly less likely to have a provincial health insurance card or to have visited another shelter in the previous 14 days. Our analysis also suggested that shelter residents who tested positive were more likely to be older and identify as racialized, but these differences were not statistically significant.

People experiencing homelessness are known to be vulnerable to COVID-19, ${ }^{4,5}$ which was confirmed by our finding of high rates of positivity in shelter residents relative to the general population. However, even within the shelter population, we found different degrees of vulnerability. Those who visited another shelter in the previous 2 weeks were less likely to test positive, perhaps because they were more mobile and therefore 


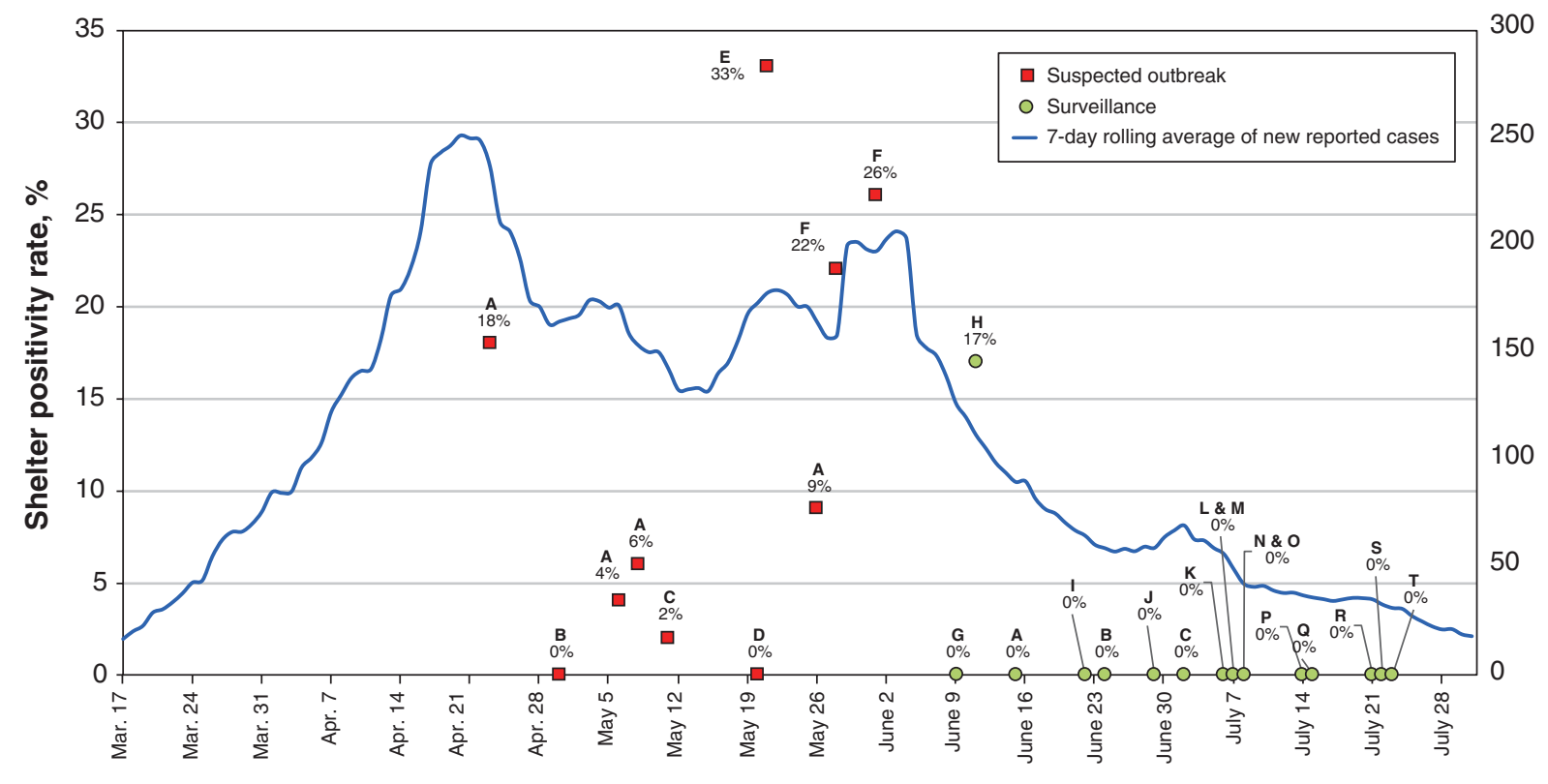

Date, 2020

Figure 1: Shelter positivity rate versus 7-day rolling average of new COVID-19 cases in Toronto. Letters refer to individual shelters.

spending more time outdoors, where there is a lower risk of transmission of SARS-CoV-2. ${ }^{19}$ In our setting, shelter residents could also have received testing in the emergency department or a COVID-19 assessment centre. Residents without a provincial health insurance card were more likely to test positive, perhaps because they delayed seeking testing for SARS-CoV-2 or because they faced more barriers to protecting themselves from infection, such as language, income or chaotic life circumstances. Racialized groups have been more severely affected by COVID-19 in Canada ${ }^{20}$ and the US. ${ }^{21,22}$ Our data suggest that homelessness and race may be intersecting factors that increase vulnerability; we support calls to address issues of structural racism at the root of poor outcomes. ${ }^{23}$

Only a handful of other studies have reported on testing for SARS-CoV-2 in homeless shelters, mostly in the US. Mosites and colleagues describe low positivity rates among shelter residents undergoing surveillance testing in Atlanta, but note positivity rates of $17 \%, 36 \%$ and $66 \%$ among residents included in outbreak testing done in Seattle, Boston and San Francisco, respectively. ${ }^{11}$ Consistent with our findings, researchers in Boston found that almost $90 \%$ of residents who tested positive in the context of an outbreak were asymptomatic. ${ }^{7}$ A preprint study of surveillance screening at 5 shelters in Rhode Island found an overall positivity rate of $12 \%$ higher than in our setting - but, like in our study, found that symptom prevalence did not vary between residents who did or did not test positive. ${ }^{12}$ A Canadian study from Hamilton, Ontario used a strategy of testing shelter residents who screened positive for symptoms and reported a very low positivity rate ${ }^{24}$ however, overall case counts have been much lower in Hamilton compared with Toronto. ${ }^{25}$

Our findings strongly support testing of asymptomatic people living in shelters where another resident has tested positive for SARS-CoV-2. We found that only 1 of 17 shelters tested for surveillance in our setting had any positive cases. This shelter had been the site of an outbreak 3 weeks prior and was also the only family shelter included in testing. Children are known to have milder presentations of COVID19 , and many studies have found asymptomatic SARS-CoV-2 infection in children. ${ }^{26}$ Our findings suggest that surveillance testing (i.e., when there is no known positive case) may detect outbreaks even when case counts are low, especially in settings with children. However, further research should evaluate other strategies for surveillance testing given scarce testing resources, including sentinel surveillance testing ${ }^{27,28}$ and the use of rapid antigen tests or saliva sampling. ${ }^{29}$

\section{Limitations}

Our study has several limitations. First, our study is based on testing done by our institution's mobile outreach team in shelters over 3 months. The 20 shelters included in our study predominantly served men and may not be representative of Toronto's shelters. A more comprehensive study would include results from people experiencing homelessness who were tested in the emergency department, COVID-19 assessment centres and by other mobile outreach teams, and would also include people sleeping outside. Second, testing was voluntary for shelter residents, which may introduce selection 


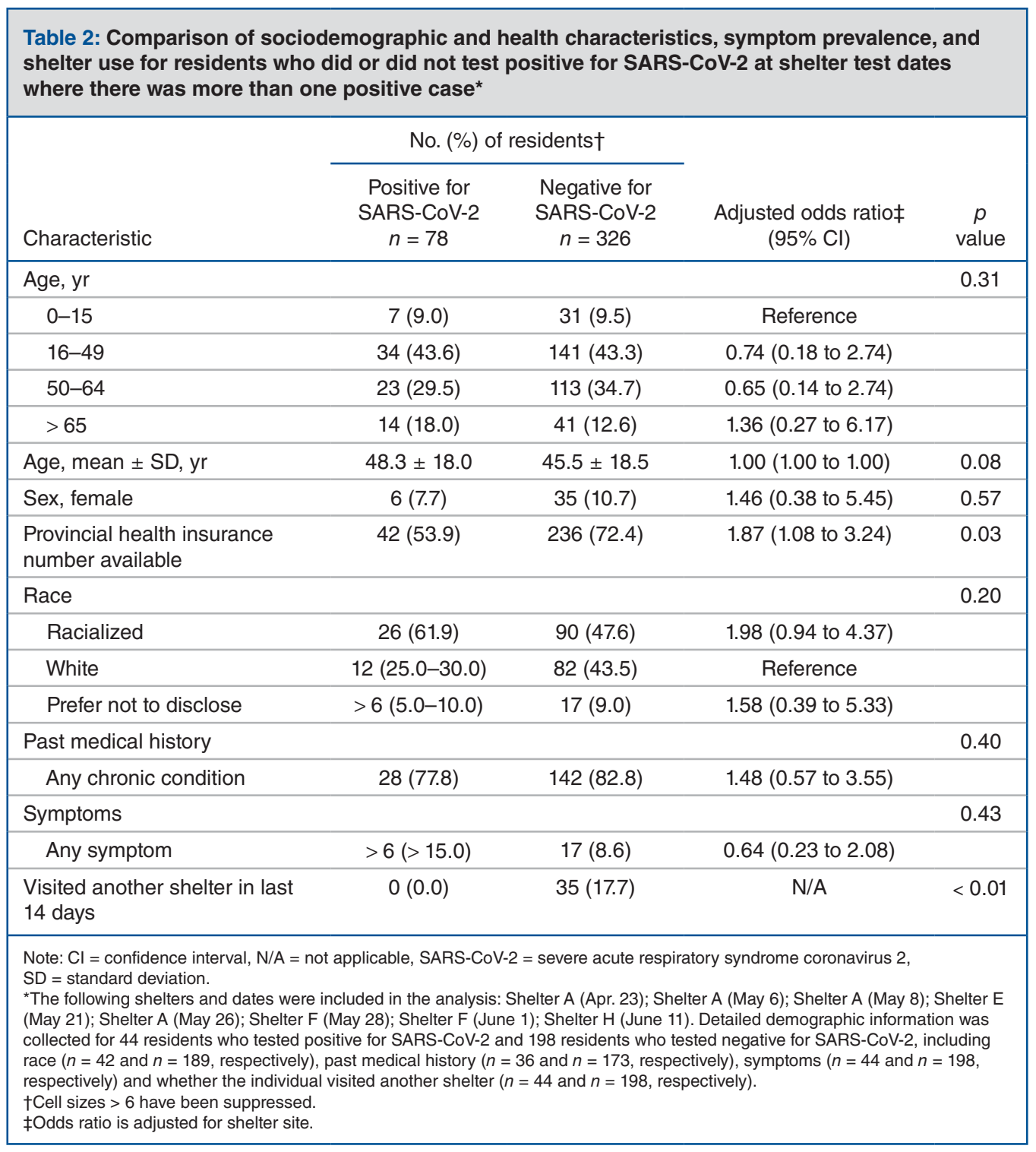

bias and influence positivity rates. Third, the precision of our estimates and our ability to detect statistically significant differences between those who did and did not test positive was limited by our sample size and the number of demographic questionnaires completed in our sample. Fourth, we did not formally assess interrater reliability with data abstraction. Fifth, the difference in positivity rates from outbreak and surveillance may be explained by the difference in community case counts and the changes to shelters to reduce transmission over the same time period. Finally, we did not engage people with lived experience of homelessness as research partners, but we hope to do so in future work.

\section{Conclusion}

In summary, we found a SARS-CoV-2 positivity rate of $14 \%$ among residents of homeless shelters tested because of a known outbreak (i.e., at least 1 known case) and a rate of $2 \%$ among residents when testing was done for surveillance (i.e., no known cases). There was no difference in health history or symptoms between residents who tested positive or negative, but there were differences related to health insurance status, use of other shelters, age and race, although the latter 2 were not statistically significant. Our findings support testing of asymptomatic shelter residents for SARS-CoV-2 when a positive case is identified. Our findings suggest that surveillance testing when there is no known positive case may detect outbreaks even when case counts are low, but further research is needed to understand how surveillance testing can be done more efficiently, given scarce testing resources. Our results also suggest that when homelessness intersects with factors such as race and health insurance status, people experience increased vulnerability to SARS-CoV-2 infection. Research and policy interventions should seek to understand and address these individual-level factors. Ultimately, solutions to the increased risk of SARS-CoV-2 transmission among people experiencing homeless need to address root causes of homelessness, including affordable housing, a living wage and adequate social supports. 


\section{References}

1. Culhane D, Henry M, Watt R, et al. 2019 AHAR: Part 1 - PIT estimates of homelessness in the U.S. HUD Exchange: 2020. Available: www.hudexchange. info/resource/5948/2019-ahar-part-1-pit-estimates-of-homelessness-in-the-us/ (accessed 2020 July 31).

2. Gaetz S, Dej E, Richter T, et al. The state of homelessness in Canada 2016. Toronto: Canadian Observatory on Homelessness Press; 2016.

3. Evidence of effect of permanent supportive housing on health. In: Permanent Supportive Housing: Evaluating the Evidence for Improving Health Outcomes Among People Experiencing Chronic Homelessness. Washington (DC): National Academies Press (US); 2018.

4. Perri M, Dosani N, Hwang SW. COVID-19 and people experiencing homelessness: challenges and mitigation strategies. CMA7 2020;192:E716-9.

5. Tsai J, Wilson M. COVID-19: A potential public health problem for homeless populations. Lancet Public Health 2020;5:e186-7.

6. Fazel S, Geddes JR, Kushel M. The health of homeless people in high-income countries: descriptive epidemiology, health consequences, and clinical and policy recommendations. Lancet 2014;384:1529-40.

7. Baggett TP, Keyes H, Sporn N, et al. Prevalence of SARS-CoV-2 infection in residents of a large homeless shelter in Boston. 7AMA 2020;323:2191-2.

8. Hsu HE, Ashe EM, Silverstein M, et al. Race/ethnicity, underlying medical conditions, homelessness, and hospitalization status of adult patients with COVID-19 at an urban safety-net medical center: Boston, Massachusetts, 2020. MMWR Morb Mortal Wkly Rep 2020;69:864-9.

9. Barocas J, Savinkina A, Linas BP. Near-term outcomes associated with COVID-19 in the United States among persons experiencing homelessness: a modelling study [preprint]. 2020 Mar. 12 Available at SSRN: https://ssrn. com/abstract=3605334 (accessed 2020 Sept. 15).

10. Wood LJ, Davies AP, Khan Z. COVID-19 precautions: easier said than done when patients are homeless. Med f Aust 2020;212:384-384.e1.

11. Mosites E, Parker EM, Clarke KEN, et al.; COVID-19 Homelessness Team. Assessment of SARS-CoV-2 infection prevalence in homeless shelters: four U.S. cities, March 27-April 15, 2020. MMWR Morb Mortal Wkly Rep 2020;69:521-2.

12. Samuels EA, Karb R, Vanjani R, et al. Congregate shelter characteristics and prevalence of asymptomatic SARS-CoV-2 [preprint]. medRxiv 2020 May 24. doi: $10.1101 / 2020.05 .21 .20108985$

13. Toronto at a glance. Toronto: City of Toronto. Available: www.toronto.ca/city -government/data-research-maps/toronto-at-a-glance/ (accessed 2020 Sept 14).

14. City of Toronto. Toronto street needs assessment 2018 results report. Toronto: Canadian Observatory on Homelessness/Homeless Hub; 2018. Available: www.homelesshub.ca/resource/toronto-street-needs-assessment-2018-results -report (accessed 2020 Sept 14).

15. INFOBulletin: COVID-19 expanding access to OHIP coverage and funding physican and hospital services for uninsured patients. Bulletin No. 4749. Toronto: Ministries of Health and Long-Term Care; 2020 Mar. 25. Available: www.health.gov.on.ca/en/pro/programs/ohip/bulletins/4000/bul4749.aspx (accessed 2021 Jan. 18)

16. COVID-19: guidance for the health sector - symptoms, screening, and testing resources. COVID-19 Guidance for the Health Sector. Toronto: Ontario Ministry of Health. Available: www.health.gov.on.ca/en/pro/programs/publichealth/ coronavirus/2019 guidance.aspx\#symptoms (accessed 2021 Jan. 15).

17. COVID-19 guidance: congregate living for vulnerable populations. Toronto: Ontario Ministry of Health; 2020. Available: http://health.gov.on.ca/en/pro/ programs/publichealth/coronavirus/docs/2019_congregate_living_guidance. pdf (accessed 2021 Jan. 15).

18. City of Toronto COVID-19 response for people experiencing homelessness [news release]. Toronto: City of Toronto: 2020 Oct. 14. Available: www. toronto.ca/news/city-of-toronto-covid-19-response-for-people-experiencing -homelessness/ (accessed 2020 Sept. 14).

19. Transmission of SARS-CoV-2: implications for infection prevention precautions [news release]. Geneva: World Health Organization; 2020 July 9. Available: www.who.int/news-room/commentaries/detail/transmission-of-sars-cov-2 -implications-for-infection-prevention-precautions (accessed 2020 Sept. 14).

20. Guttmann A, Gandhi S, Wanigaratne S, et al. COVID-19 in immigrants, refugees and other newcomers in Ontario: characteristics of those tested and those confirmed positive, as of June 13, 2020. Toronto: ICES; 2020. Available: www.ices.on.ca/Publications/Atlases-and-Reports/2020/COVID-19-in -Immigrants-Refugees-and-Other-Newcomers-in-Ontario (accessed 2020 Sept. 14).

21. Webb Hooper M, Nápoles AM, Pérez-Stable EJ. COVID-19 and racial/ethnic disparities. FAMA 2020;323:2466-7.

22. Price-Haywood EG, Burton J, Fort D, et al. Hospitalization and mortality among black patients and white patients with COVID-19. $N$ Engl 7 Med 2020;382:2534-43.

23. Egede LE, Walker RJ. Structural racism, social risk factors, and COVID-19: a dangerous convergence for black Americans. N Engl f Med 2020;383:e77.
24. Bodkin C, Mokashi V, Beal K, et al. Pandemic planning in homeless shelters: a pilot study of a COVID-19 testing and support program to mitigate the risk of COVID-19 outbreaks in congregate settings. Clin Infect Dis 2020 June 8 [Epub ahead of print]. doi: 10.1093/cid/ciaa743.

25. ICES COVID-19 Dashboard. Toronto: ICES; updated 2021 Feb. 4. Available: https://www.ices.on.ca/DAS/AHRQ/COVID-19-Dashboard (accessed 2021 Jan. 15).

26. Al-Sadeq DW, Nasrallah GK. The incidence of the novel coronavirus SARSCoV-2 among asymptomatic patients: a systematic review. Int $\mathcal{Z}$ Infect Dis 2020;98:372-80.

27. Bond A. COVID-19 response framework for people experiencing homelessness. Toronto: Canadian Observatory on Homelessness/Homeless Hub; 2020. Available: www.homelesshub.ca/resource/covid-19-response-framework-peo ple-experiencing-homelessness (accessed 2020 Aug. 29)

28. Geard N, McVernon J, Gibney K. What is sentinel surveillance and how might it help in the fight against coronavirus? The Conversation [Toronto] 2020 Apr. 30. Available: https://theconversation.com/what-is-sentinel-surveillance -and-how-might-it-help-in-the-fight-against-coronavirus-136845 (accessed 2020 Sept. 8).

29. Wyllie AL, Fournier J, Casanovas-Massana A, et al. Saliva or nasopharyngeal swab specimens for detection of SARS-CoV-2. N Engl 7 Med 2020;383:1283-6.

Affiliations: MAP Centre for Urban Health Solutions (Kiran, Craig-Neil, Wang, Snider, Hwang), and Department of Family and Community Medicine (Kiran, Das, Rosenthal), and Department of Emergency Medicine (Lockwood, Snider), and Department of Medicine (Hwang), St. Michael's Hospital, University of Toronto; Department of Family and Community Medicine (Kiran, Das, Rosenthal), and Institute of Health Policy, Management and Evaluation (Kiran, Snider), and Division of Emergency Medicine (Lockwood, Snider), and MD program (Nathanielsz), and Division of General Internal Medicine (Hwang), Department of Medicine, Faculty of Medicine, University of Toronto, Toronto, Ont.

Contributors: Tara Kiran conceived the study. Tara Kiran, Amy CraigNeil, Paul Das, Joel Lockwood, Ri Wang, Esther Rosenthal, Carolyn Snider and Stephen Hwang designed the study. Amy Craig-Neil and Nikki Nathanielsz collected the data, and Amy Craig-Neil, Nikki Nathanielsz and Ri Wang conducted the analysis. All authors helped interpret the data. Tara Kiran drafted the manuscript and all authors critically reviewed it. All authors read and approved the final manuscript.

Funding: This study was supported by a grant from the St. Michael's Hospital Foundation. The study sponsor had no role in study design, data collection, analysis, interpretation of data, manuscript preparation or the decision to submit for publication.

Content licence: This is an Open Access article distributed in accordance with the terms of the Creative Commons Attribution (CC BY-NC-ND 4.0) licence, which permits use, distribution and reproduction in any medium, provided that the original publication is properly cited, the use is noncommercial (i.e., research or educational use), and no modifications or adaptations are made. See: https://creativecommons.org/licenses/ by-nc-nd/4.0/

Data sharing: After deidentification, individual participant data that underlie the results reported in this article can be made available to researchers who provide a methodologically sound proposal. Requests should be directed to the corresponding author and will be granted between 3 and 36 months after article publication.

Acknowledgements: The authors thank Linh Luong and Tadios Tibebu, who spent many hours extracting data from paper charts. The authors also thank the many people involved in supporting the mobile outreach testing, particularly Dana Whitham, Nicole Gichuru, Chantel Marshall and Linda Jackson, whose leadership and guidance made the outreach possible. The authors would like to especially acknowledge the daily hard work by staff at the COVID-19 Assessment Centre at St. Michael's Hospital, Sherbourne Health, and the shelter sites.

Supplemental information: For reviewer comments and the original submission of this manuscript, please see www.cmajopen.ca/content/9/1/ E302/suppl/DC1. 